\title{
Development of an electrochemical CCL17/TARC biosensor towards rapid triage and monitoring of classic Hodgkin Lymphoma
}

Christopher Rinaldi ${ }^{*}$, Damion K. Corrigan ${ }^{2} \ddagger$, Lynn Dennany ${ }^{1} \ddagger$, Ruth F. Jarrett ${ }^{3}$, Annette Lake $^{3}$, Matthew J. Baker ${ }^{4}$

1 WestCHEM, Department of Pure and Applied Chemistry, Technology and Innovation Centre, University of Strathclyde, 99 George Street, Glasgow, G1 1RD, UK.

2 Department of Biomedical Engineering, University of Strathclyde, 40 George Street, Glasgow, G1 1QE, UK. 3 MRC-University of Glasgow Centre for Virus Research, 464 Bearsden Rd, Bearsden, Glasgow, G61 1QH, UK.

4 DXCOVER Ltd., University of Strathclyde, Technology and Innovation Centre, 99 George Street, Glasgow, G1 1RD, UK

*Corresponding author: Dr Christopher Rinaldi, The Technology \& Innovation Centre, University of Strathclyde, 99 George Street, Glasgow, G1 1RD, E-mail: christopher.rinaldi@strath.ac.uk, Phone: +44 (0) 141 4447000

Supporting Information Table of Contents

Supplementary Methods..

Cleaning.....

Immobilisation

Reproducibility Study.

Scanning Electron \& Atomic Force Microscopy.

Patient Information ..Table S1

Electrochemical Cleaning Figure $\mathrm{S} 1$

SAM Characterisation: Probability Distribution Figure S2

Reproducibility Study.

Figure S3

Evaluation of Electrode Surfaces.

.Figures S4 \& S5 


\section{Supplementary Methods}

Cleaning. Working electrodes were chemically cleaned in hot piranha solution comprised of sulphuric acid and $30 \%$ hydrogen peroxide in water (3:1 ratio) for 10 minutes to remove organic contaminants. Electrodes were subsequently mechanically cleaned with $0.05 \mu \mathrm{m}$ alumina MicroPolish powder on a Microcloth pad for 5 minutes and sonicated in DI water for 15 minutes to remove alumina particles. Thereafter, working electrodes were electrochemically cleaned in $0.5 \mathrm{M}$ sulphuric acid in DI water by applying cyclic potential sweeps between vertices of $-0.2 \mathrm{~V}$ and $+1.5 \mathrm{~V}$ with a scan rate of $100 \mathrm{mV} / \mathrm{s}$ for 20 consecutive scans.

Immobilisation. Firstly, $40 \mathrm{mM}$ Sulfo-LC-SPDP in DI water and $200 \mu \mathrm{g} / \mathrm{ml}$ primary CCL17/TARC antibodies (2:1 ratio) in $1 \times$ PBS were agitated at room temperature for 1 hour. Secondly, $150 \mathrm{mM}$ DTT in $1 \times$ PBS was introduced for 45 minutes. Thirdly, $3 \mathrm{KDa}$ centrifugal filters were used to remove unreacted Sulfo-LC-SPDP and DTT prior to assay deposition. Lastly, assay filtrate was reconstituted in $1 \times \mathrm{PBS}$, pipetted on to working electrodes and incubated in a humidity chamber at $4{ }^{\circ} \mathrm{C}$ for $\sim 15$ hours. Working electrodes were rinsed with $1 \times$ PBS to remove excess antibody solution. Rinse procedures were repeated after respective assay depositions to remove unbound molecules. Remaining bare electrodes were blocked with $1 \mathrm{mM} \mathrm{MCH}$ in $1 \times$ PBS and $5 \mathrm{mM}$ TCEP for 30 minutes to prevent non-specific binding. Electrodes were incubated with $25 \mu \mathrm{L}$ of target CCL17 antigen $(24-50,000 \mathrm{pg} / \mathrm{ml})$ in $1 \times$ PBS or clinical cHL samples, diluted ten-fold in $1 \times$ PBS, for 1 hour at room temperature. Electrodes were incubated with $25 \mathrm{uL}$ of $200 \mathrm{ng} / \mathrm{mL}$ biotinylated CCL17/TARC secondary antibody solution in $1 \times$ PBS for 1 hour at room temperature. $25 \mu \mathrm{L}$ of $\times 40$ diluted streptavidin-conjugated horseradish-peroxidase in $1 \times$ PBS were pipetted on working electrodes out of direct sunlight for 20 minutes. Electrodes were immersed in $500 \mu \mathrm{L}$ substrate solution comprised of equal volumes of stabilised 3,3',5,5'-Tetramethylbenzidine (TMB) and stabilised hydrogen peroxide for 20 minutes.

Reproducibility Study. A reproducibility study was conducted on functionalised electrodes with EIS to evaluate inter-assay variability of SAM formation prior to antigen exposure. EIS was conducted with electrodes immersed in a solution of $10 \mathrm{mM}$ ferri/ferro potassium cyanide in $1 \times$ PBS following respective assay depositions. EIS measurements utilised previously specified parameters and considered five functionalised working electrodes per measurement day over a 7-day period.

Scanning Electron \& Atomic Force Microscopy. Scanning electron microscopy (SEM) experiments were performed with a JEOL JSM-IT100 InTouchScope configured in high vacuum mode with an acceleration voltage of 10-20 kV. Images were acquired from three random sites on working electrodes at x1700-10,000 magnification at a working distance of 10-14 mm to provide accurate representations of substrates. Atomic force microscopy (AFM) experiments were conducted with the Asylum Research MFP-3D instrument with triangular AFM cantilevers configured in contact mode. Images were acquired from three random sites on working electrodes to provide accurate topographical information on substrates. 
Patient Information

\begin{tabular}{|c|c|c|c|}
\hline Patient Number & Patient ID & Sample ID & Clinical Serum Sample Type \\
\hline 1 & 10089 & 53810 & Healthy Volunteer \\
\hline 2 & 10154 & 54371 & Healthy Volunteer \\
\hline 3 & 10157 & 54490 & Healthy Volunteer \\
\hline 4 & 10174 & 54597 & Healthy Volunteer \\
\hline 5 & 10280 & 57477 & Healthy Volunteer \\
\hline 6 & 10581 & 63016 & Pre-Treatment HL \\
\hline 7 & 10639 & 63552 & Pre-Treatment HL \\
\hline 8 & 36530 & 96349 & Pre-Treatment HL \\
\hline 9 & 10578 & 63159 & Pre-Treatment HL \\
\hline 10 & 11783 & 66671 & Pre-Treatment HL \\
\hline 11 & 36547 & 96677 & Pre-Treatment HL \\
\hline 12 & 36564 & 97029 & Pre-Treatment HL \\
\hline 13 & 36578 & 97964 & Pre-Treatment HL \\
\hline 14 & 36521 & 97279 & Pre-Treatment HL \\
\hline 15 & 36558 & 97793 & Pre-Treatment HL \\
\hline 16 & 36524 & 96224 & Pre-Treatment HL \\
\hline 17 & 11810 & 66845 & Pre-Treatment HL \\
\hline 18 & 36536 & 97183 & Pre-Treatment HL \\
\hline 19 & 36604 & 98242 & Pre-Treatment HL \\
\hline 20 & 36567 & 97994 & Pre-Treatment HL \\
\hline 21 & 10622 & 64197 & Pre-Treatment HL \\
\hline 22 & 36566 & 97348 & Pre-Treatment HL \\
\hline 23 & 36514 & 96186 & Pre-Treatment HL \\
\hline 24 & 36529 & 96900 & Pre-Treatment HL \\
\hline 25 & 36577 & 98218 & Pre-Treatment HL \\
\hline 26 & 36586 & 97695 & Pre-Treatment HL \\
\hline 27 & 36554 & 97506 & Pre-Treatment HL \\
\hline 28 & 36512 & 96925 & Pre-Treatment HL \\
\hline 29 & 36532 & 96318 & Pre-Treatment HL \\
\hline 30 & 36515 & 96992 & Pre-Treatment HL \\
\hline 31 & 36540 & 97333 & Pre-Treatment HL \\
\hline 32 & 36548 & 96793 & Pre-Treatment HL \\
\hline 33 & 36519 & 96259 & Pre-Treatment HL \\
\hline 34 & 36575 & 97488 & Pre-Treatment HL \\
\hline 35 & 36565 & 97165 & Pre-Treatment HL \\
\hline 36 & 36574 & 97481 & Pre-Treatment HL \\
\hline 37 & 36520 & 97384 & Pre-Treatment HL \\
\hline 38 & 36526 & 97553 & Pre-Treatment HL \\
\hline 39 & 36539 & 97859 & Pre-Treatment HL \\
\hline 40 & 9269 & 51351 & Pre-Treatment HL \\
\hline 41 & 11696 & 65873 & Pre-Treatment HL \\
\hline 42 & 11906 & 67794 & Pre-Treatment HL \\
\hline 43 & 11854 & 67181 & Pre-Treatment HL \\
\hline 44 & 9366 & 52145 & Pre-Treatment HL \\
\hline 45 & 10374 & 56421 & Pre-Treatment HL \\
\hline 46 & 36513 & 96115 & Pre-Treatment HL \\
\hline 47 & 36580 & 97544 & Pre-Treatment HL \\
\hline 48 & 36565 & 97209 & On-Treatment HL \\
\hline 49 & 36513 & 96159 & On-Treatment HL \\
\hline 50 & 36539 & 97959 & On-Treatment HL \\
\hline 51 & 36520 & 97469 & On-Treatment HL \\
\hline 52 & 36519 & 96340 & On-Treatment HL \\
\hline 53 & 36514 & 96279 & On-Treatment HL \\
\hline 54 & 36574 & 97682 & On-Treatment HL \\
\hline
\end{tabular}

Table S1: Overview of clinical details for tested classical Hodgkin lymphoma patient serum samples. 


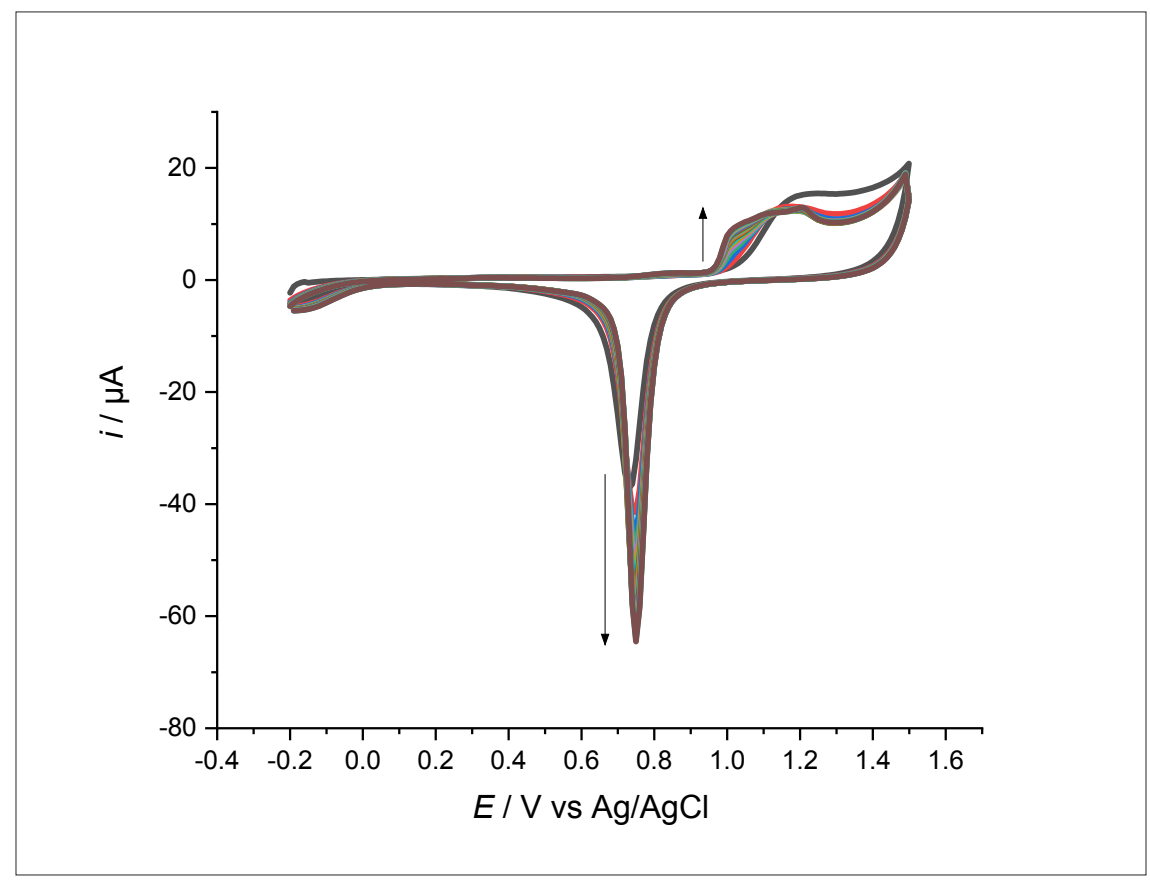

Figure S1: Electrochemical cleaning of working electrodes in $0.5 \mathrm{M} \mathrm{H}_{2} \mathrm{SO}_{4}$ from $-0.2 \mathrm{~V}$ to $1.5 \mathrm{~V}$ for 20 scans $(\mathrm{n}=3)$ prior to assay functionalisation procedures. Arrows indicate consecutive increases in both oxidation and reduction peaks with successive sweeps.

Working electrodes subjected to electrochemical cleaning procedures observed consecutive increases in one broad $I_{p a}$ at $+1.0 \mathrm{~V}$ and one sharp $I_{p c}$ at $\sim 0.75 \mathrm{~V}$ characteristic of clean gold, depicted in Figure S1. The first scan measured $I_{p c}=-37.3 \mu \mathrm{A} \pm 5.57$ at $E_{p c}=0.729 \mathrm{~V}$ and increased with successive scans to $I_{p c}=-64.5 \mu \mathrm{A} \pm 5.72$ at $E_{p c}=0.749 \mathrm{~V}$ by the twentieth scan, indicative of progressive gold oxide removal at electrode surfaces. The calculated electrochemical surface area (ECSA) of cleaned working electrodes $\left(0.1209 \mathrm{~cm}^{-2} \pm 0.0043\right)$ was greater than the geometrical electrode area $\left(0.0314 \mathrm{~cm}^{-2}\right)$, further indicative of clean and electrochemically active surfaces. The ECSA of working electrodes was obtained by calculating the charge associated with reduction of gold oxide, through integration of the reductive peak associated with the $20^{\text {th }}$ scan and dividing by the scan rate, and then subsequently dividing by the capacitance for clean gold surfaces, assumed to be $390 \mu \mathrm{Ccm}^{-2}$. 


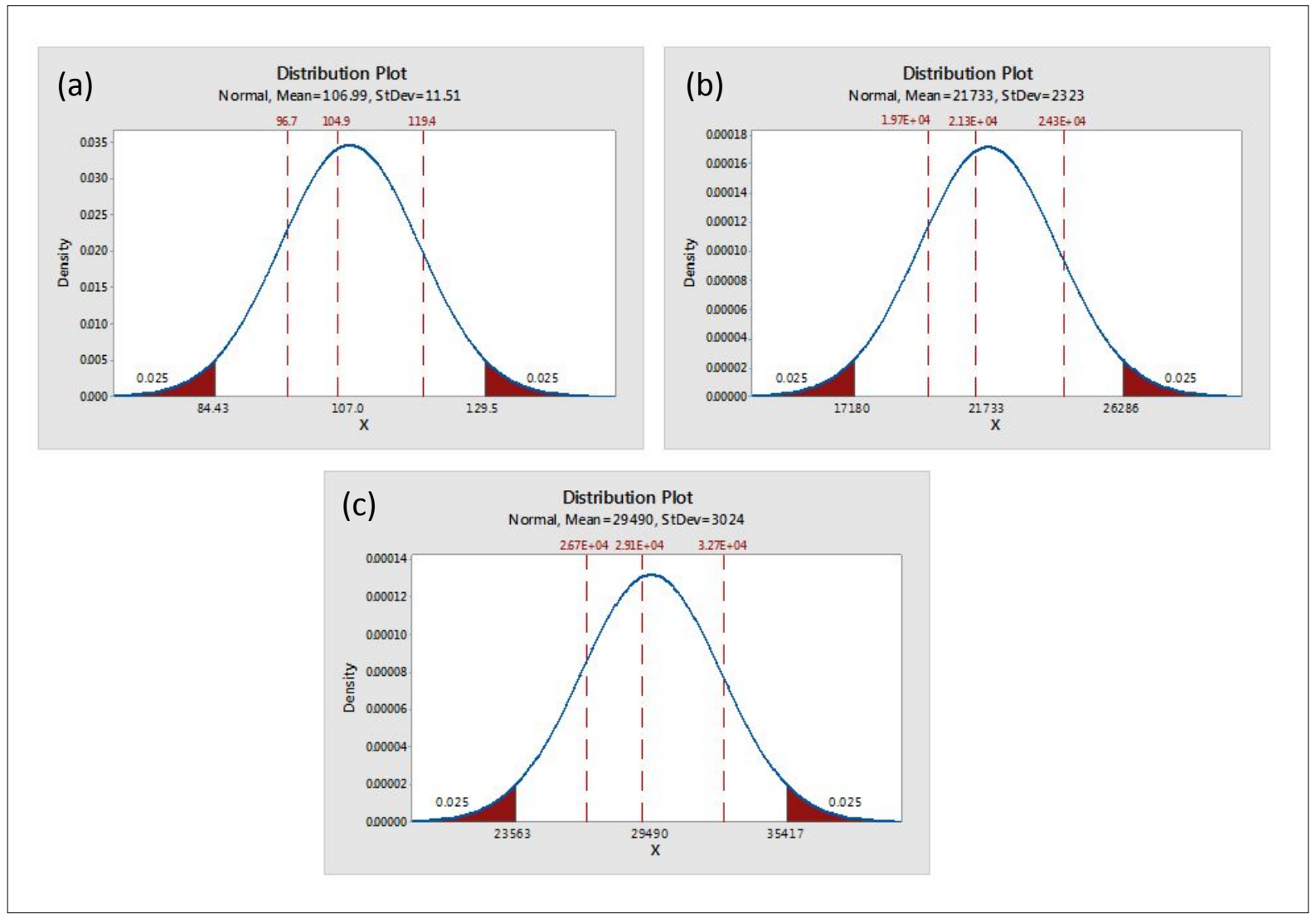

Figure S2: Probability distribution plots showing the variation in $\mathrm{R}_{\mathrm{ct}}$ for (a) bare gold electrodes and (b) Sulfo-LC-SPDP primary CCL17/TARC antibody and (c) MCH immobilisation steps at a specified 95\% confidence interval. Red dashed lines indicate the absolute $\mathrm{R}_{\mathrm{ct}}$ value for individual measurements for respective assay depositions. 


\section{Reproducibility Study}

Electrode functionalisation varied considerably over the 7-day measurement period, with charge transfer resistance, $\mathrm{R}_{\mathrm{ct}}=$ $6,107.06 \Omega \pm 8,345.59$ associated with Sulfo-LC-SPDP/antibody depositions and $\mathrm{R}_{\mathrm{ct}}=13,947.57 \Omega \pm 10,074.48$ for electrode backfilling procedures, indicated in Figure S3.

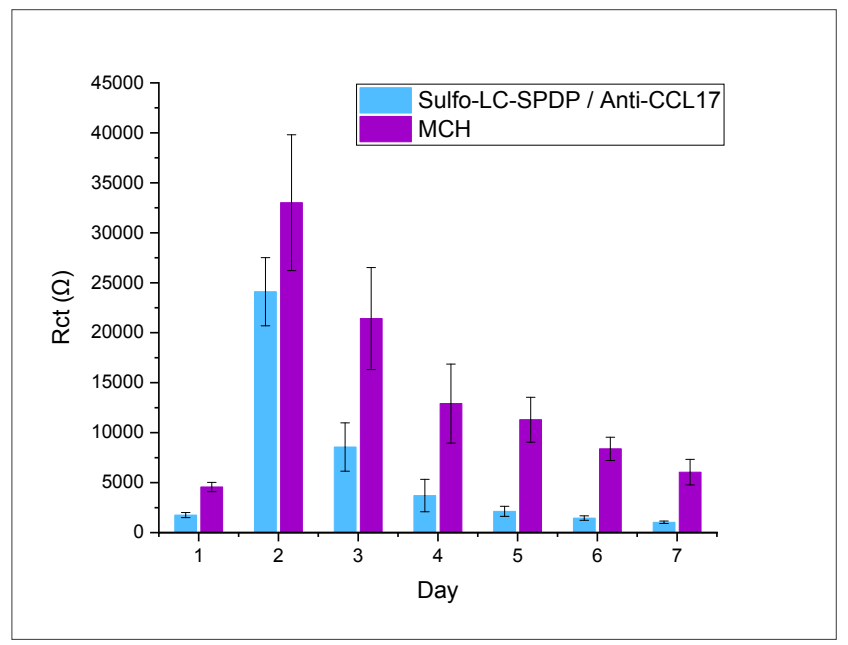

Figure S3: Reproducibility plot showing charge transfer resistance, $\mathrm{R}_{\mathrm{ct}}$, associated with different assay functionalisation deposits between electrodes per day $(n=5)$ and on different days over the course of a 7-day time period. Results represent mean $\pm \mathrm{SD}$ and $\mathrm{R}_{\mathrm{ct}}$ values calculated through circuit fitting with Randles equivalent circuit and Levenberg-Marquardt model. 


\section{Evaluation of Electrode Surfaces}

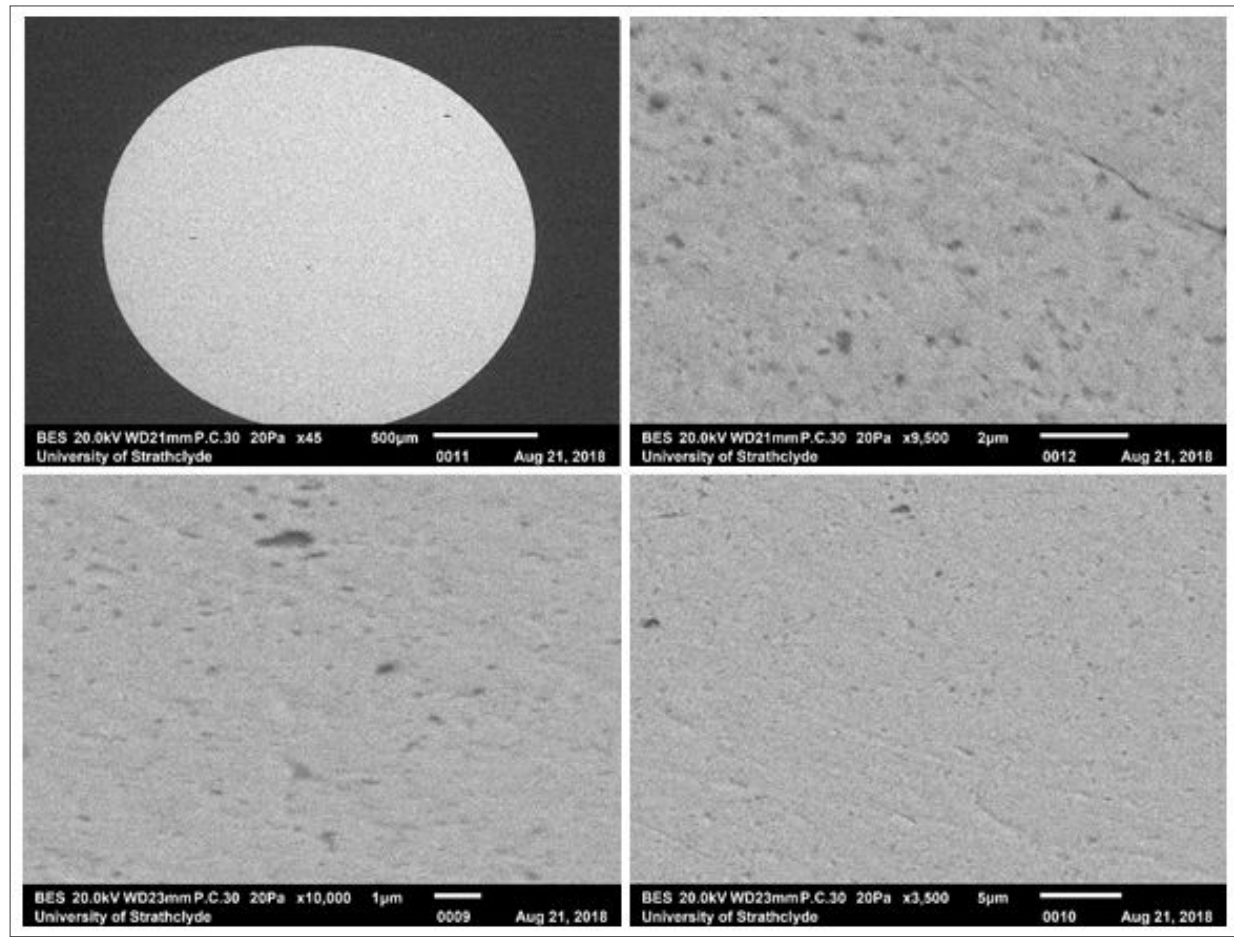

Figure S4: SEM images of bare gold CH Instruments CHI101 macro polycrystalline electrodes.

Inspection of clean electrode surfaces with SEM reveals a degree of surface roughness with visible scratches and pitting across electrodes, likely attributable to repeated mechanical polishing procedures, as indicated in Figure S4.

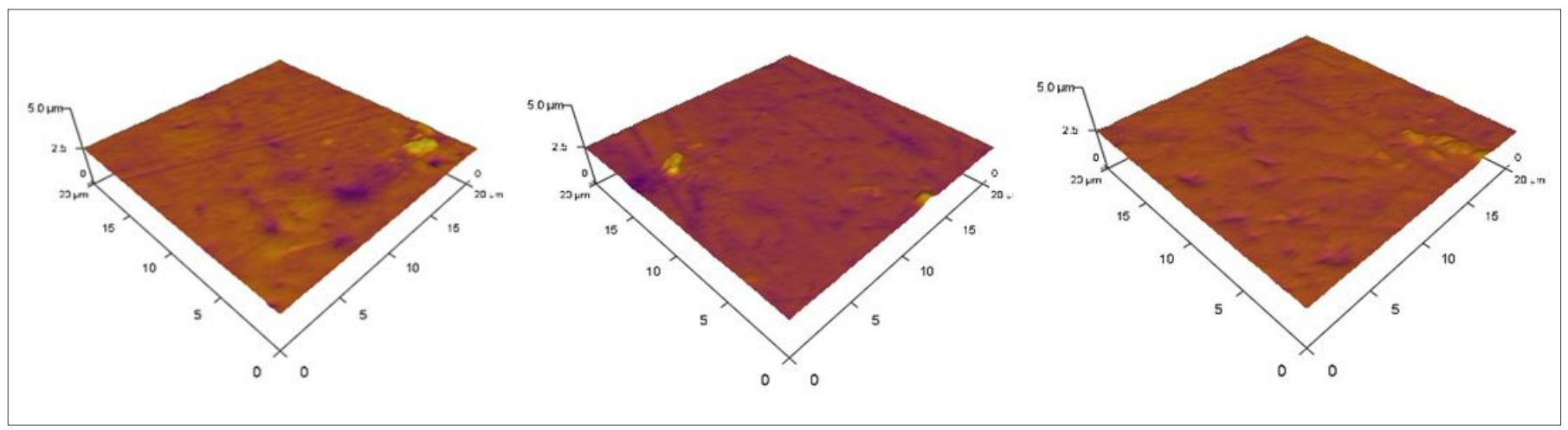

Figure S5: AFM images of bare gold CH Instruments CHI101 macro polycrystalline electrodes.

Inspection of clean electrode surfaces with AFM further confirmed evidence of deviations in surface morphology and superficial scratches likely from repeated mechanical electrode polishing, as indicated in Figure S5. Nevertheless, AFM measurements demonstrated relatively smooth electrode surfaces, with root mean squared surface roughness values of $19.43 \mathrm{~nm}, 14.32 \mathrm{~nm}$ and $26.01 \mathrm{~nm}$, calculated from cross-sections of all three electrode samples respectively. Furthermore, peak morphological features for all three electrode samples measured $354.94 \mathrm{~nm}, 382.71 \mathrm{~nm}$ and $276.83 \mathrm{~nm}$ respectively, reiterating the presence of relatively smooth electrode surfaces. 